\title{
Diffraction of Gaussian Laser Beams by a Semi-Infinite Plane
}

\author{
J. E. Pearson,* T. C. McGiri, S. Kurtin, and A. Yariv $\dagger$ \\ California Institute of Technology, Pasadena, California 91109
}

(Received 12 April 1969)

\begin{abstract}
Theoretical and experimental aspects of the diffraction of gaussian laser beams by the straight edge bounding an opaque plane are investigated. Theoretical analysis is based upon the Kirchhoff scalar wave theory in the Fresnel limit, assuming an incident electromagnetic field having spatial amplitude and phase variation appropriate to a fundamental-mode gaussian beam. Experimental observation consisting of irradiance as a function of position is in good agreement with this theory. Both theoretical and experimental results are found to depend strongly on gaussian-beam parameters.

INDEX HEADINGS: Diffraction; Lasers.
\end{abstract}

The diffraction of electromagnetic radiation by material objects is, in principle, a well-understood area of classical physics. Although attempts to formulate a rigorous theoretical approach to this phenomenon suffer from certain fundamental inadequacies, ${ }^{1-4}$ the approximations of Kirchhoff and Sommerfeld are valid in most regions of interest ${ }^{5-7}$; they have been confirmed to excellent precision for an incident plane wave with microwave $e^{8-11}$ and noncoherent optical sources, ${ }^{12,13}$ and with electron beams. ${ }^{14}$

Many useful optical sources do not produce beams that can be approximated by a uniform plane wave. Therefore, it is of interest to consider modifications to the plane-wave diffraction theory appropriate to nonuniform beams. The advent of laser sources evokes new tests of diffraction theory and suggests a consideration of the approximations appropriate to this special case. The unique phase and spatial-amplitude variation which is characteristic of lasers makes them ideal sources for diffraction experiments dealing with beamsize effects. In addition, the high irradiance and lowbeam divergences available greatly simplify the experimental apparatus needed to perform high-accuracy measurements.

* Fannie and John Hertz Foundation doctoral fellow.

$\uparrow$ Work supported by the Office of Naval Research through Contract Nonr (220-50).

$1 \mathrm{~B}$. B. Baker and E. T. Copson, The Mathematical Theory of Huygens' Principle (Oxford University Press, New York, 1930), 2nd ed.

${ }^{2}$ A. Sommerfeld, Optics (Academic Press Inc., New York, 1954).

${ }^{3}$ E. Marom, J. Opt. Soc. Am. 57, 1390 (1967).

- M. Born and E. Wolf, Principles of Optics (Pergamon Press, Inc., New York, 1964), 3rd ed.

' G. Kirchhoff, Vorlesungen Über Math. Physik. 2 (Optik, Leipzig, 1891).

${ }^{6}$ E. Wolf and E. W. Marchand, J. Opt. Soc. Am. 54, 587 (1964).

${ }_{7}$ E. W. Marchand and E. Wolf, J. Opt. Soc. Am. 56, 1712 (1966).

${ }^{8}$ C. W. Horton and R. B. Watson, J. Appl. Phys. 21, 16 (1950).

${ }^{\circ}$ R. D. Kodis, J. Appl. Phys. 23, 249 (1952).

${ }^{20}$ B. N. Harden, Proc. Inst. Elec. Engrs. Pt. III 99, 229 (1952).

11 R. V. Row, J. Appl. Phys. 24, 1448 (1953).

${ }^{12}$ K. L. McDonald and F. S. Harris, Jr., J. Opt. Soc. Am. 42, 321 (1952).

${ }_{23}$ J. D. Barnett and F. S. Harris, Jr., J. Opt. Soc. Am. 52, 536 (1962).

is J. Komrska, V. Drahos, and A. Delong, Opt. Acta 11, 145 (1964).
In this paper we examine theoretical and experimental aspects of the diffraction of finite-size laser beams by the straight edge bounding an opaque plane. The resulting irradiance patterns differ significantly, but in a predictable manner, from the plane-wave case. In addition to providing quantitative experimental data in agreement with the scalar diffraction theory, this paper will show that even the qualitative character of the observed diffraction pattern depends on beam size and divergence angle.

\section{THEORETICAL ANALYSIS}

\section{A. Kirchhoff-Fresnel Formulation}

The theoretical problem of an adequate formulation of the generalized diffraction problem has been discussed elsewhere. ${ }^{1-3}$ In this paper we employ the well-established Kirchhoff-Fresnel scalar analysis, ${ }^{4-7}$ using an incident electromagnetic field which is similar to that of a TEM T00 $_{\text {laser mode. }}$

The Kirchhoff-Fresnel formulation expresses the amplitude of the scalar field behind an aperture in terms of the integral

$$
\begin{aligned}
U(\bar{r})=\frac{-1}{4 \pi} \int_{\sigma}\left[U_{\mathrm{inc}}\left(\bar{r}^{\prime}\right) \frac{\partial}{\partial n}\left(\frac{e^{i k\left|\bar{r}-\bar{r}^{\prime}\right|}}{\left|\vec{r}-\bar{r}^{\prime}\right|}\right)\right. \\
-\frac{\left.e^{i k\left|\bar{r}-\bar{r}^{\prime}\right|} \frac{\partial}{\left|\tilde{r}-\bar{r}^{\prime}\right|} \frac{\partial n}{\partial n} U_{\mathrm{inc}}\left(\bar{r}^{\prime}\right)\right] d s^{\prime}}{}
\end{aligned}
$$

where $U_{\text {ino }}\left(\bar{r}^{\prime}\right)$ is the scalar amplitude of the incident field and the integral is taken over the diffracting aperture (see Fig. 1). A more rigorous solution also requires that $U_{\text {ino }}$ be a solution to the wave equation

$$
\left(\nabla^{2}+k^{2}\right) U_{\text {inc }}=0 .
$$

Following the accepted theory of optical resonators, ${ }^{15-18}$ we take $U_{\text {ino }}$ as the field distribution of a

${ }^{15}$ G. D. Boyd and J. P. Gordon, Bell System Tech. J. 40, 489 (1961).

${ }^{16}$ H. Kogelnik, Appl. Opt. 4, 1562 (1965).

${ }^{17}$ G. D. Boyd and H. Kogelnik, Bell System Tech. J. 41, 1347 (1962).

${ }^{18}$ A. G. Fox and T. Li, Bell System Tech. J. 40, 453 (1961). 
fundamental-mode gaussian beam given by ${ }^{15,16}$

$$
\begin{aligned}
U_{\text {ino }}(\bar{r}) & =\frac{\omega_{0}}{\omega(z)} \exp \left\{i[k z+\beta(z)]-r^{2}\left[\frac{1}{\omega^{2}(z)}+\frac{i k}{2 R(z)}\right]\right\} \\
& =\psi(\bar{r}) e^{-i \zeta(\mathrm{r})} .
\end{aligned}
$$

The incident beam is assumed to propagate in the $z$ direction; $r=\left(x^{2}+y^{2}\right)^{\frac{1}{2}}$ is the distance from the $z$ axis, $k=2 \pi / \lambda$ is the propagation constant in the medium, and $\beta(z)$ is a phase shift due to the beam geometry. The radius of the beam (i.e., the spot size) is $\omega(z)$ and the radius of curvature of the phase front is $R(z) . \omega, R$, and $\beta$ are given by ${ }^{16}$

$$
\begin{aligned}
& \omega^{2}(z)=\omega_{0}{ }^{2}\left[1+\left(\frac{2 z}{b}\right)^{2}\right] \\
& R(z)=z\left[1+\left(\frac{b}{2 z}\right)^{2}\right] \\
& \beta(z)=\tan ^{-1}\left(\frac{2 z}{b}\right),
\end{aligned}
$$

where $b=2 \pi \omega_{0}^{2} / \lambda$ is the beam confocal parameter and $z$ is measured from the beam waist where $\omega=\omega_{0}$. As the gaussian beam propagates and expands, its transverse amplitude variation remains gaussian. The $\frac{1}{2}$ angle of divergence of the beam is given by $\tan \delta=\omega(z) / z$, which for $z \gg b / 2$ is

$$
\tan \delta \simeq \frac{2 \omega_{0}}{b}=[2 \lambda / b \pi]^{\frac{1}{2}}=\frac{\lambda}{\pi \omega_{0}}
$$

The function given by Eq. (3) is an approximate solution to Eq. (2) in the limiting case in which $d^{2} \psi / d z^{2}$ can be neglected. It is valid even when $k^{2}$ is a quadratic function of $r$,

$$
k^{2}=k_{0}^{2}\left(1-\frac{k_{2}}{k_{0}} r^{2}\right)
$$

and thus is a good description of the beam from a laser, since the laser gain is known to vary quadratically with $r^{19,20}$

Two difficulties arise in using Eq. (3) as a description of an experimental laser beam. First, it is necessary to determine the parameters in Eq. (3) such as $\omega_{0}$ (or $b$ ) and the distance from the beam waist to the plane of interest (the diffraction edge), $z=Z_{01}$. This is discussed below. Second, there is usually some mixture of higherorder modes present in laser beams. These difficulties are not serious, however, and do not affect the validity of the theory; they merely complicate its experimental verification.

${ }^{19}$ W. R. Bennett, Jr. and J. W. Knutson, Jr., Bull. Am. Phys. Soc. 9, 500 (1964).

${ }_{20}$ W. R. Bennett, Jr., Appl. Opt., Suppl. 2,3 (1965).

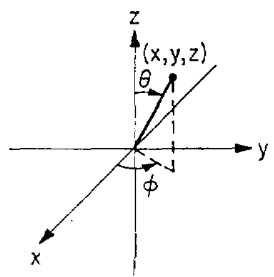

(a)

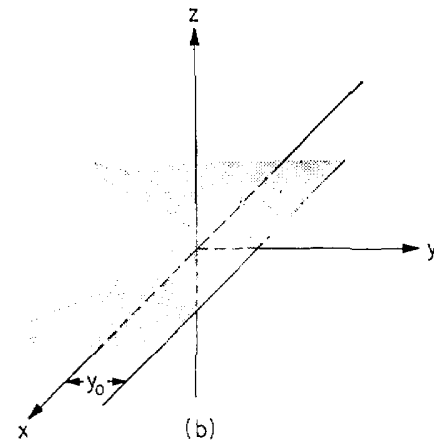

FIG. 1. Coordinate system for diffraction at a straight edge. (a) Definition of the angles $\theta$ and $\phi$ used in Eq. (8). (b) Definition of the aperture $\sigma: \sigma \equiv\left(y>y_{0}\right.$, all $\left.x\right)$. The incident beam travels toward positive $z$ and has its maximum at $x=y=0$.

Substituting Eq. (3) in Eq. (1), taking $\sigma$ as a semiinfinite half-plane, and going to the Fresnel limit, we find the field behind the aperture,

$$
\begin{aligned}
& U(\bar{r})= {\left[2 /\left(1+\xi_{0}^{2}\right)\right]^{\frac{1}{2}}\left[e^{i k r} / 8 r(C G)^{\frac{1}{2}}\right] } \\
& \times \exp \left[\frac{H^{2}}{4 G}-\frac{k^{2}}{4 C} \sin ^{2} \theta \cos ^{2} \phi\right] \\
& \times\left\{\left[\frac{L}{2 G}+P\right] \operatorname{erfc}(F)+\pi^{-\frac{1}{2}}\left[\frac{F L}{G}+Q\right] \exp \left(-F^{2}\right)\right\}
\end{aligned}
$$

where the conventional polar coordinates are defined as in Fig. $1 ; y_{0}$ is the location of the edge with respect to the incident beam (centered about $x=y=0$ ) and

$$
\begin{aligned}
& \xi_{0}=\frac{2 Z_{01}}{b} \quad \alpha=\frac{k}{b\left(1+\xi_{0}^{2}\right)}\left(1-i \xi_{0}\right) \\
& A=\frac{2 k}{b^{2}} \frac{2 \xi_{0}+i\left(1-\xi_{0}^{2}\right)}{\left(1+\xi_{0}^{2}\right)^{2}} \\
& B=i k\left(1+\frac{z}{r}\right)-\frac{2\left(\xi_{0}+i\right)}{b\left(1+\xi_{0}^{2}\right)} \\
& C=\alpha+i k\left(\sin ^{2} \theta \cos ^{2} \phi-1\right) / 2 r \\
& D=\alpha+i k\left(\sin ^{2} \theta \sin ^{2} \phi-1\right) / 2 r \\
& F=G^{\frac{1}{2}}\left(y_{0}+\frac{H}{2 G}\right) \\
& G=D+\left(k^{2} / 16 C r^{2}\right) \sin ^{4} \theta \sin ^{2} 2 \phi \\
& H=i k \sin \theta \sin ^{2}+\left(k^{2} / 4 C r\right) \sin ^{3} \theta \cos \phi \sin 2 \phi \\
& L=A\left(1-\frac{k^{2}}{16 C{ }^{2} r^{2}} \sin ^{4} \theta \sin ^{2} 2 \phi\right) \\
& M=\frac{-A k^{2}}{4 C^{2} r} \sin ^{3} \cos ^{2} \sin ^{2} \phi
\end{aligned}
$$




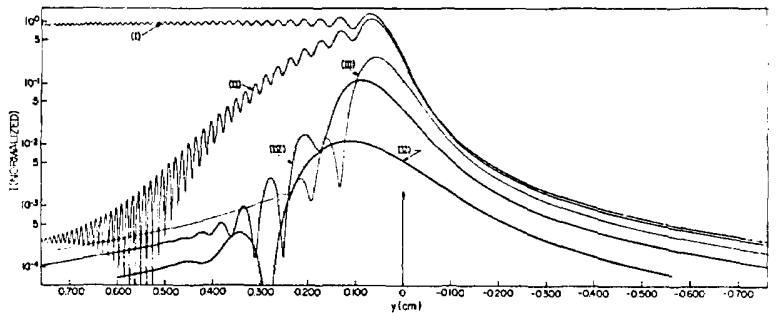

FIG. 2. Theoretical normalized relative irradiance $I$ vs distance. ${ }^{21}$ The curves are calculated from Eq. (8) and are labeled corresponding to the gaussian-beam parameters given in Table $I$. The arrow at $y=0$ defines the location of the geometrical edge.

$$
\begin{aligned}
& N=B+\frac{A}{2 C}\left(1-\frac{k^{2} \sin ^{2} \theta \cos ^{2} \phi}{2 C}\right) \\
& P=N-(M H / 2 G)+\left(L H^{2} / 4 G^{2}\right) \\
& Q=(M / \sqrt{ } G)-\left(L H / G^{3}\right) .
\end{aligned}
$$

The result given by Eq. (8) is normalized to unit incident flux

$$
\int_{-\infty}^{\infty} \int_{-\infty}^{\infty}|U(\bar{r})|^{2}{ }_{z=0} d x d y=1 .
$$

To normalize to unit peak irradiance, $U(r)$ as given by Eq. (8) should be multiplied by $\left[1+\xi_{0}^{2}\right]^{\sharp} / \sqrt{2}$.

From Eq. (8), the diffracted irradiance distribution has been evaluated to high precision for various cases of interest using digital-computer techniques. For a range of incident-beam parameters (shown in Table I), Fig. 2 illustrates the results of the computations and compares the quasi-plane-wave case (Case I) with three finite-beam cases. ${ }^{21}$ Note that each curve is characterized by both the confocal parameter $b$ and the distance $Z_{01}$. From these two quantities, both the spot size and divergence angle of the incident beam are determined. All curves in Fig. 2 are computed for

$\lambda=6328 \AA$ (He-Ne laser), $y_{0}=0, \quad z=100 \mathrm{~cm}, \quad \theta \simeq 0$ and

$\phi=\pi / 2$ (light region) or $\phi=3 \pi / 2$ (shadow region).

TABLE I. Gaussian-beam parameters for diffraction patterns. (Theoretical analysis)

\begin{tabular}{lcccc}
\hline Case & $b=2 L_{01}(\mathrm{~cm})$ & $Z_{01}(\mathrm{~cm})$ & $\begin{array}{c}\text { Location of } \\
\text { first maximum } \\
(\mathrm{cm})\end{array}$ & $\begin{array}{c}\text { Normalized } \\
\text { irradiance } \\
\text { at } y=0\end{array}$ \\
\hline I & 34.30 & 3320 & 0.0686 & 0.236 \\
II & 1552.0 & 1480 & 0.0686 & 0.225 \\
III & 189.3 & 101.0 & 0.0584 & 0.110 \\
IV & 34.30 & 57.00 & 0.0889 & 0.0355 \\
V & 15.0 & 15.00 & 0.1143 & 0.00529 \\
\hline
\end{tabular}

21 The detail and rapid oscillations in the curves of Fig. 2 can best be appreciated by referring to the expanded-scale figures in the experimental section of this paper.

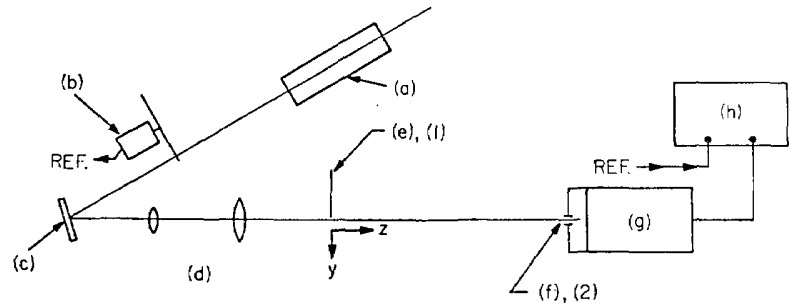

FIG. 3. Schematic of experimental irradiance-measuring apparatus. (a) $\mathrm{He}-\mathrm{Ne}$ laser. (b) Chopper. (c) Mirror. (d) $5 \mathrm{X}$ telescope. (e) Diffracting edge. (f) Scanning slit. (g) Slit and detector assembly. (h) Lock-in amplifier. (1) and (2) are locations 1 and 2, respectively, as used in Eqs. (12)-(15) and are $100 \mathrm{~cm}$ apart.

Because each incident beam is normalized to unit peak irradiance at the edge, the significance of beam size and rate of divergence on diffraction phenomena is immediately clear from examination of Fig. 2. The behavior of the diffraction pattern in the light region is easily understood by considering the Young theory of diffraction ${ }^{1}$ along with the classical Huygens construction. This interpretation is discussed below.

\section{B. Interpretation of Theoretical Results}

Using the physical interpretation of diffraction given by Huygens and Young, ${ }^{1,22}$ we can take the total irradiance of the field beyond the screen as

$I(\bar{r})=\left|\psi_{\text {ino }}(\bar{r})+\psi_{\text {diff }}(\bar{r})\right|^{2}$; geometrical light region $\left|\psi_{\mathrm{diff}}(\bar{r})\right|^{2}$; geometrical shadow region.

Thus, if the amplitude of the diffracted field is approximately equal to that of the incident field, large spatial irradiance fluctuations may be observed in the light region.

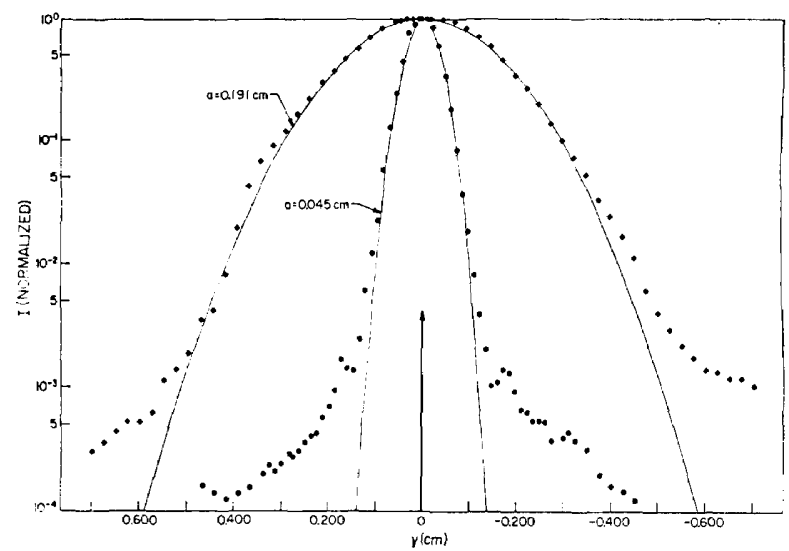

FIG. 4. Normalized beam-irradiance profiles measured at the location of the diffracting edge $(z=0$, location 1$)$ and compared to a best-fit gaussian envelope. $\$=$ experimental, large beam $(a=0.191 \mathrm{~cm}) ; \bullet=$ experimental, small beam $(a=0.045 \mathrm{~cm})$; $\underline{-}=$ gaussian fit, $y=\exp \left[-y^{2} / a^{2}\right]$. The arrow at $y=0$ defines the location of the geometrical edge.

${ }^{22}$ See K. Miyamoto and E. Wolf, J. Opt. Soc. Am. 52, 615, 626 (1962) for a mathematical formulation of Young's theory. 
Applying this interpretation to the curves of Fig. 2, we see that in the case of the quasi-plane wave (Case I), the amplitude of the diffracted field is, at all points in space, much less than the incident field and only small irradiance fluctuations occur. For the finite beams, however, the amplitude of the incident beam may drop sufficiently rapidly with increasing distance from the diffracting edge that the diffracted and incident fields have roughly the same amplitude over a finite spatial region. In this region, the resultant irradiance as given by Eq. (9) is driven toward zero at every point where the phase difference between the diffracted and incident beam is $180^{\circ}$. This phenomenon is observed in Cases II-V of Fig. 2.

Note that the symmetry of the irradiance distribution about the geometrical edge increases as the size of the incident beam is reduced (i.e., $\omega_{0}$ is reduced) and hence the secondary wavelets from the edge of the diffracting screen play a larger role in the irradiance distribution. ${ }^{23}$ It is also interesting to note the change of height of the first maximum in the light region, as well as its location and the value of the irradiance at the geometrical shadow boundary, as the parameters of the incident beam are varied.

\section{EXPERIMENTATION}

\section{A. Experimental Apparatus and Techniques}

The measurement technique employed in this series of experiments is shown schematically in Fig. 3. A $\mathrm{He}-\mathrm{Ne}$ laser having an unpolarized power output of approximately $1 \mathrm{~mW}$ in the TEMI ${ }_{00 q}$ mode was used as a source. The beam was expanded with a $5 \mathrm{X}$ telescope. Irradiances were measured with lock-in techniques with a $15 \mu \mathrm{m} \times 0.6-\mathrm{cm}$ scanning slit and an RCA 925 vacuum photodiode. For convenience, the diffracting edge was a razor blade. Bennett and $\mathrm{Harris}^{13}$ have shown that neither the conductivity nor the sharpness of the edge has any important effect on the irradiance distribution of the diffraction pattern.

Before we could measure the diffraction patterns, we had to determine the effective beam parameters of the incident laser beam. At a fixed z, Eq. (3) gives the normalized transverse irradiance variation as

$$
I=|U(\bar{r})|^{2}=\mid \exp \left[-i k r^{2} /\left.2 q(z)\right|^{2}=\exp \left[-k r^{2} / L(z)\right],\right.
$$

where

$$
\frac{1}{q(z)}=\frac{1}{R(z)}-\frac{i}{L(z)}, \quad \text { and } \quad L(z)=\frac{\pi \omega^{2}(z)}{\lambda} .
$$

${ }^{23}$ From a superficial examination of Fig. 2, it appears that diffraction in the limit of small beam sizes is not purely an edge effect as predicted by Huygens' principle. It is possible to show, however, (see Refs. $6,7,22$ ) that, even for small incident beams, diffraction is an edge effect. The size and rate of expansion of the beam play ever-increasing roles in determining the exact nature of the diffraction pattern as the beam size is reduced.

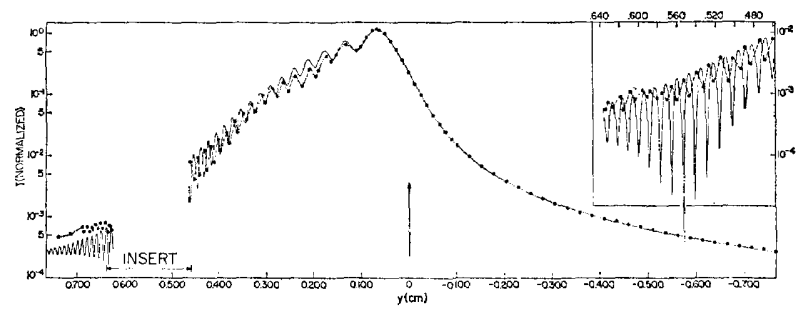

FIG. 5. Experimental data and theoretical diffraction pattern for large incident beam $\left(a_{1}=0.191 \mathrm{~cm}\right.$ and Case II of Table I and Fig. 2). $I$ is normalized relative irradiance as defined in Eq. (16). The arrow at $y=0$ defines the location of the geometrical edge.

$R(z)$ is the radius of curvature of the beam and $\omega(z)$ is the spot radius [see Eqs. (4)-(6)].

For homogeneous media, such as free space, Kogelnik $^{16,24}$ has shown that

$$
q_{2}=q_{1}+Z_{12}
$$

where $q_{2}$ and $q_{1}$ are the beam parameters at $z=z_{2}$ and $z=z_{1}$, respectively, and $Z_{12}=\left(z_{2}-z_{1}\right)$.

Defining $L(z)$ by

$$
L(z)=\frac{\pi \omega^{2}(z)}{\lambda}=\frac{2 \pi a^{2}(z)}{\lambda},
$$

where $a$ is the gaussian-irradiance half-width at $z$, we can use Eq. (11) and Eq. (12) to solve for $R_{1}$ when $L_{1}$, $L_{2}$, and $Z_{12}$ are known. That is, we must solve the equation

$$
\begin{aligned}
& R_{1}{ }^{4}\left(L_{1}{ }^{2}-L_{1} L_{2}+Z_{12}{ }^{2}\right)+R_{1}{ }^{3}\left(2 L_{1}{ }^{2} Z_{12}\right) \\
& +R_{1}{ }^{2}\left(L_{1}^{4}-L_{1}{ }^{3} L_{2}+2 L_{1}{ }^{2} Z_{12}{ }^{2}\right) \\
& \quad+R_{1}\left(2 L_{1}{ }^{4} Z_{12}\right)+L_{1}{ }^{4} Z_{12}{ }^{2}=0 .
\end{aligned}
$$

The positive, real solution to Eq. (14) is then used with $L_{1}$ to find the effective beam parameters $L_{01}$ (and thus $b$ ) and $Z_{01}$ at location 1 . We find

$$
\begin{aligned}
& L_{01}=\frac{R_{1}^{2} L_{1}}{R_{1}^{2}+L_{1}^{2}}=\frac{b}{2} \\
& Z_{01}=\frac{R_{1} L_{1}^{2}}{R_{1}^{2}+L_{1}^{2}} .
\end{aligned}
$$

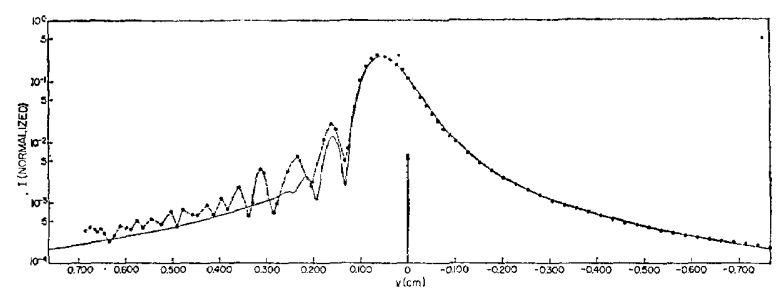

FIG. 6. Experimental data and theoretical diffraction pattern for small incident beam $\left(a_{1}=0.045 \mathrm{~cm}\right.$ and Case III of Table I and Fig. 2). $I$ is normalized relative irradiance as defined in $\mathrm{Eq}$. (16). The arrow at $y=0$ defines the location of the geometrical edge.

${ }^{24}$ H. Kogelnik, Bell System Tech. J. 44, 455 (1965). 

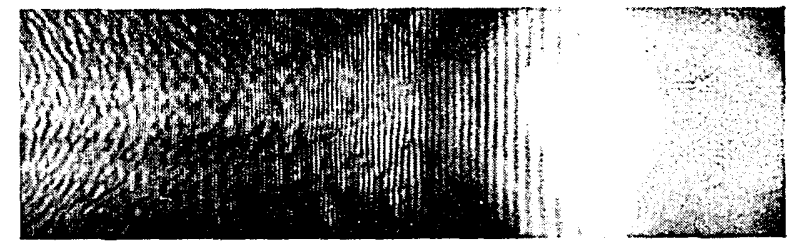

(a)

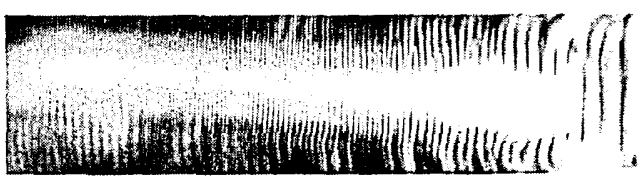

(b)

FIG. 7. Photographs of the fringes corresponding to the experimental data of Figs. 4 and 5: (a) large beam (Fig. 5); (b) small beam (Fig. 6). The dynamic range has been compressed; see text.

The parameters for the theoretical curves in Figs. 5 and 6 were determined by measuring the irradiance profile of the laser beam at locations 1 and 2 as defined in Fig. 3. Gaussian curves fitted to the irradiance profiles resulted in the values of $a$ necessary to determine $b$ and $Z_{01}$. Typical irradiance profiles at the edge location (location 1, $z=0$ ) plotted together with fitted gaussian curves are shown in Fig. 4. Equation (8) was then used to generate the theoretical diffraction patterns.

Figure 4 shows that the incident-beam profile differs considerably from a pure gaussian envelope after the irradiance has dropped by 2 or 3 orders of magnitude. The longer tail on the actual beam profile is due to a combination of inhomogeneities in the laser mirrors and higher-order modes present in the laser beam. The difficulties that these higher modes introduce into a comparison of theory with experiment are discussed below.

The diffracting edge was aligned with the center of the incident gaussian beam by the following procedure:

(1) The scanning slit was positioned in the center (maximum value) of the incident laser beam. This defines the position $x=0, y=0, z=100 \mathrm{~cm}$ as in Fig. 1,

(2) The straight edge was inserted into the beam until the measured normalized irradiance fell to the normalized value predicted in the theoretical calculations. Thus the curves in Figs. 5 and 6 are necessarily matched at $y=0$. Because the theoretical calculations assume unit irradiance at the location of the edge $(z=0)$, the experimental curves are also normalized to the maximum of the beam at $z=0$. Thus we have

$$
\begin{array}{r}
{\left[I_{\text {mens }}(y=0)\right]_{\text {norm }}=\frac{I_{\text {meas }}(y=0, z=100 \mathrm{~cm})}{I_{\text {meas }}(y=0, z=0)}} \\
=I_{\text {theor. }}(y=0, z=100 \mathrm{~cm}) .
\end{array}
$$

The procedure we used here removes the ambiguity of edge placement relative to the center of the incident beam and eliminates the otherwise necessary technique of employing a one-adjustable-parameter fit of experimental data to theoretical calculations.

Following this alignment procedure, we measured irradiance as a function of distance from the known reference point $(x=0, y=0, z=100 \mathrm{~cm})$. Irradiance was measured along a line perpendicular to the axis of the incident beam rather than on a circle of fixed radius. The difference between these two procedures is negligible for the small values of $y$ and the large value of $z$ appropriate to this experiment.

\section{B. Measurements}

Figures 5 and 6 show experimental data and theoretical curves calculated for the incident beams of Fig. 4. Experimental data points were taken at or near the locations of maxima and minima. A smooth curve, shown dotted, was drawn through these points. Note the wide dynamic range, $10^{4}$ of irradiance, achievable as a result of employing a laser source. The theoretical diffraction patterns of Figs. 5 and 6 are reproductions of curves (II) and (III), respectively, of Fig. 2.

In addition to these quantitative measurements, we determined the fringe positions photographically by replacing the scanning slit with a lensless, focal-planeshutter, 35-mm camera loaded with EG \& G XR film. This film is a three-layer dye-transfer material that is capable of recording spatial irradiance variations having dynamic irradiance ranges up to eight orders of magnitude. Prints (with compressed dynamic irradiance range) of the diffraction patterns are shown in Fig. 7 for both the large-beam and small-beam cases. The well-defined interference fringes that correspond to the data presented in Figs. 5 and 6 are clearly visible.

\section{Errors and Discussion}

In view of the inherent high accuracy of lock-in techniques and the accuracy of the technique used to position the edge in the beam, the principal experimental errors are due to (1) finite scanning-slit size, $.0015 \times .6 \mathrm{~cm}$; (2) difficulty of aligning the scanning slit parallel to the diffracting edge; (3) higher-order modes and amplitude fluctuations of the irradiance of the laser source.

The first two of these account for the shallowness of the undulation of the measured data compared to the theoretical beam and tend to cause the averaging over successive fringes that is evident in Figs. 5 and 6. Reference to Fig. 7, however, shows that the length of the slit $(0.6 \mathrm{~cm})$ did not introduce any error due to curvature of the fringes. Thus the experimental technique averages the irradiance in the $x$ direction. The normalizing technique then compensates for the finite slit length. 
The third source of error is the most serious, introducing uncertainty into the determination of relative irradiance as well as into the determination of equivalent beam parameters for theoretical calculations. Systematic qualitative disagreement between theory and experiment at large distances from the geometrical edge can be understood in terms of the deviation of the actual incident-beam profiles from those used in the theoretical calculation. This deviation is caused by the presence of higher-order modes than $\mathrm{TEM}_{00 q}$ in the experimental beam. The physical interpretation discussed in Sec. IIB is useful here. For example, in the experimental data of Figs. 5 and 6, spatial irradiance variations can be seen on top of the measured incidentbeam profiles, as shown in Fig. 4 (including the nongaussian tail). These variations arise from the interference of the incident beam with secondary Huygens wavelets propagating from the edge.

The quantitative disagreement between theory and experiment evident in Figs. 5 and 6 is believed to result from amplitude fluctuations of the incident beam and from our inability to determine accurately the spot radius $a$. The inaccuracy in the determination of $a$ occured because the incident beam was not a pure gaussian. This inaccuracy causes the disagreement between theory and experiment in the location of fringe maxima and minima. Theoretical diffraction patterns computed from Eq. (8) are quite sensitive to the two values of $a$ used to find $Z_{01}$ and $b$.

Taking into account all known sources of error, we estimate our maximum error to be approximately $10 \%$ in the light region and $5 \%$ in the shadow region, in both relative irradiance and in location of the fringes. Agreement between theory and experiment in the light region is good. In the geometrical shadow, the agreement is excellent in view of the wide dynamic range of measured irradiances and indicates that the diffracted wave is independent of the detailed nature of the incident beam.

\section{CONCLUSION}

From these experiments we may conclude that the scalar Kirchhoff-Fresnel formulation of diffraction theory is valid to a good approximation for the diffraction of laser beams of finite size. The theory predicts the magnitude of the diffraction irradiances as well as the location of the fringes. Implicit in this formulation, however, are beam-size effects that must be taken into account when unexpanded laser beams are used. A simple physical model based on the Huygens construction and the Young theory of diffraction is often useful in understanding diffraction phenomena, particularly for small beams. 